\title{
Aharonov-Bohm oscillations in the presence of strong spin-orbit interactions
}

\author{
Boris Grbić* ${ }^{*}$ Renaud Leturcq*, Thomas Ihn*, Klaus Ensslin*, Dirk Reuter ${ }^{+}$, and Andreas D. Wieck ${ }^{+}$ \\ ${ }^{*}$ Solid State Physics Laboratory, ETH Zurich, 8093 Zurich, Switzerland \\ ${ }^{+}$Angewandte Festkörperphysik, Ruhr-Universität Bochum, 44780 Bochum, Germany
}

\begin{abstract}
We have measured highly visible Aharonov-Bohm (AB) oscillations in a ring structure defined by local anodic oxidation on a p-type GaAs heterostructure with strong spin-orbit interactions. Clear beating patterns observed in the raw data can be interpreted in terms of a spin geometric phase. Besides $h / e$ oscillations, we resolve the contributions from the second harmonic of $\mathrm{AB}$ oscillations and also find a beating in these $h / 2 e$ oscillations. A resistance minimum at $B=0 \mathrm{~T}$, present in all gate configurations, is the signature of destructive interference of the spins propagating along time-reversed paths.
\end{abstract}

Interference phenomena with particles have challenged physicists since the foundation of quantum mechanics. A charged particle traversing a ring-like mesoscopic structure in the presence of an external magnetic flux $\Phi$ acquires a quantum mechanical phase. The interference phenomenon based on this phase is known as the Aharonov-Bohm (AB) effect [1], and manifests itself in oscillations of the resistance of the mesoscopic ring with a period of $\Phi_{0}=h / e$, where $\Phi_{0}$ is the flux quantum. The Aharonov-Bohm phase was later recognized as a special case of the geometric phase [2, 3] acquired by the orbital wave function of a charged particle encircling a magnetic flux line.

The particle's spin can acquire an additional geometric phase in systems with spin-orbit interactions (SOI) [4, 5, 6]. The investigation of this spin-orbit (SO) induced phase in a solid-state environment is currently the subject of intensive experimental work [7, $8,2,10,11,12]$. The common point of these experiments is the investigation of electronic transport in ring-like structures defined on two-dimensional (2D) semiconducting systems with strong SOI. Electrons in InAs were investigated in a ring sample with time dependent fluctuations [7], as well as in a ring side coupled to a wire [9]. An experiment on holes in GaAs [8] showed B-periodic oscillations with a relative amplitude $\Delta R / R<10^{-3}$. These observations [7, 8] were analyzed with Fourier transforms and interpreted as a manifestation of Berry's phase. Further studies on electrons in a HgTe ring [10] and in an InGaAs ring network [11] were discussed in the framework of the AharonovCasher effect.

In systems with strong SOI, an inhomogeneous, momentum dependent intrinsic magnetic field $\mathbf{B}_{\text {int }}$, perpendicular to the particle's momentum, is present in the reference frame of the moving carrier [13]. The total magnetic field seen by the carrier is therefore $\mathbf{B}_{\text {tot }}=$ $\mathbf{B}_{\text {ext }}+\mathbf{B}_{\text {int }}$, where $\mathbf{B}_{\text {ext }}$ is the external magnetic field perpendicular to the $2 \mathrm{D}$ system and $\mathbf{B}_{\mathbf{i n t}}$ is the intrinsic magnetic field in the plane of the $2 \mathrm{D}$ system present in the moving reference frame (right inset Fig. 1(a)). The particle's spin precesses around $\mathbf{B}_{\text {tot }}$ and accumulates an additional geometric phase upon cyclic evolution.

Effects of the geometric phases are most prominently expressed in the adiabatic limit, when the precession fre- quency of the spin around the local field $\mathbf{B}_{\text {tot }}$ is much faster than the orbital frequency of the charged particle carrying the spin [4]. In this limit the ring can be considered to consist of two uncoupled types of carriers with opposite spins [14]. The total accumulated phase, composed of the $\mathrm{AB}$ phase and the $\mathrm{SO}$ induced geometric phase, is different for the two spin species, $\phi_{t o t}=\phi_{A B} \pm \phi_{S O}$, and the magnetoresistance of the ring is obtained as the superposition of the oscillatory contributions from the two spin species. Such a superposition is predicted to produce complex, beating-like magnetoresistance oscillations with nodes developing at particular values of the external Bfield, where the oscillations from the two spin-species have opposite phases [5]. Both $h / e$ and $h / 2 e$ peaks in the Fourier spectrum of the magnetoresistance oscillations are predicted to be split in the presence of strong SOI [5, 15].

The interpretation of the split Fourier signal in Ref. 7] has been challenged [16]. The data on p-GaAs rings 8] stirred an intense discussion [17]. Our raw data directly displays a beating of the $h / e$ Aharonov-Bohm oscillations. No Fourier transform is required to verify this effect. As additional evidence we directly measure a beating of the $h / 2 e$ oscillations and a pronounced and persistent zero field magnetoresistance minimum due to destructive interference of time-reversed paths.

The sample was fabricated by atomic force microscope (AFM) oxidation lithography on a p-type carbon doped (100) GaAs heterostructure, with a shallow twodimensional hole gas (2DHG) located $45 \mathrm{~nm}$ below the surface [18]. An AFM micrograph of the ring structure is shown in the inset of Fig. 1(a). The average radius of the circular orbit is $420 \mathrm{~nm}$, and the lithographic width of the arms is $190 \mathrm{~nm}$, corresponding to an electronic width of $60-70 \mathrm{~nm}$. The hole density in an unpatterned sample is $3.8 \times 10^{11} \mathrm{~cm}^{-2}$ and the mobility is $200000 \mathrm{~cm}^{2} / \mathrm{Vs}$ at a temperature of $60 \mathrm{mK}$. Therefore the Fermi wavelength is about $40 \mathrm{~nm}$, and the mean free path is $2 \mu \mathrm{m}$. Since the circumference of the ring is around $2.5 \mu \mathrm{m}$, the transport through the ring is quasiballistic. From the temperature dependence of the $\mathrm{AB}$ oscillations we extract the phase coherence length of the holes to be $L_{\varphi}=2 \mu \mathrm{m}$ at a base temperature of $T=60 \mathrm{mK}$.

The presence of strong spin-orbit interactions in the 
heterostructure is demonstrated by a simultaneous observation of the beating in Shubnikov-de Haas (SdH) oscillations and a weak anti-localization dip in the measured magnetoresistance of the Hall bar fabricated on the same wafer [19]. In p-type GaAs heterostuctures, Rashba SOI is typically dominant over the Dresselhaus SOI [20]. The densities $\mathrm{N}_{1}=1.35 \times 10^{11} \mathrm{~cm}^{-2}$ and $\mathrm{N}_{2}=$ $2.45 \times 10^{11} \mathrm{~cm}^{-2}$ of the spin-split subbands, deduced from $\mathrm{SdH}$ oscillations, allow us to estimate the strength of the Rashba spin-orbit interaction $\Delta_{S O} \approx 0.8 \mathrm{meV}$ assuming a cubic wave-vector dependence [13]. Due to the large effective mass of the holes, the Fermi energy in the system, $E_{F}=2.5 \mathrm{meV}$, is much smaller than that in electron systems with the same density. The large ratio $\Delta_{S O} / E_{F} \approx 30 \%$ documents the presence of strong SOI.

We have measured the four-terminal resistance of the ring in a ${ }^{3} \mathrm{He} /{ }^{4} \mathrm{He}$ dilution refrigerator at a base temperature of about $60 \mathrm{mK}$ with lock-in techniques. A low ac current of $2 \mathrm{nA}$ and $31 \mathrm{~Hz}$ frequency was applied in order to prevent sample heating.

Fig. 1(a) shows the magnetoresistance of the ring (fast oscillating curve, red online). The low-frequency background resistance is indicated by a smooth curve (blue online). The observed Aharonov-Bohm (AB) oscillations with a period of $7.7 \mathrm{mT}$ (frequency $130 \mathrm{~T}^{-1}$ ) correspond to a radius of the holes' orbit of $415 \mathrm{~nm}$, in excellent agreement with the lithographic size of the ring. The peak-to-peak amplitude of $\sim 200 \Omega$ on a background of about $6 \mathrm{k} \Omega$ corresponds to a visibility larger than $3 \%$. We restrict the measurements of the $\mathrm{AB}$ oscillations to magnetic fields in the range from $-0.2 \mathrm{~T}$ to $0.2 \mathrm{~T}$ in order to prevent their mixing with $\mathrm{SdH}$ oscillations, which start to develop above $0.2 \mathrm{~T}$. Throughout all measurements quantum point contact gates $3,4,5$ and 6 are kept at the same values. Plunger gates pg1 and pg2 are set to $\mathrm{V}_{p g 1}=-145 \mathrm{mV}$ and $\mathrm{V}_{p g 2}=-95 \mathrm{mV}$ in the measurements presented in Fig. 1(a).

After subtracting the low-frequency background from the raw data, a clear beating pattern is revealed in the AB oscillations with a well defined node at $\sim 115 \mathrm{mT}$ [Fig. 1(b)], where a phase jump of $\pi$ occurs [arrow in Fig. 2(c)]. The position of the beating node indicates the presence of two oscillation frequencies differing by $1 / 0.115 \approx 9 \mathrm{~T}^{-1}$.

The Fourier spectrum of the $\mathrm{AB}$ oscillations, taken in the symmetric magnetic field range $(-0.2 \mathrm{~T}, 0.2 \mathrm{~T})$, reveals an $h / e$ peak around $130 \mathrm{~T}^{-1}$ [Fig. 1(c)]. Zooming in on the $h / e$ peak, a splitting into 3 peaks at the frequencies $127 \mathrm{~T}^{-1}, 136 \mathrm{~T}^{-1}$ and $143 \mathrm{~T}^{-1}$ is seen. We have carefully checked that this splitting is genuine to the experimental data and not a result of the finite data range, by reproducing it with different window functions for the Fourier transform. The differences of the oscillation frequencies agree with that anticipated from the position of the beating node in the raw data.

In contrast to the $h / e$-periodic $\mathrm{AB}$ oscillations, which are very sensitive to phase changes in the ring arms, Altshuler-Aronov-Spivak (AAS) $h / 2 e$ oscillations, orig-
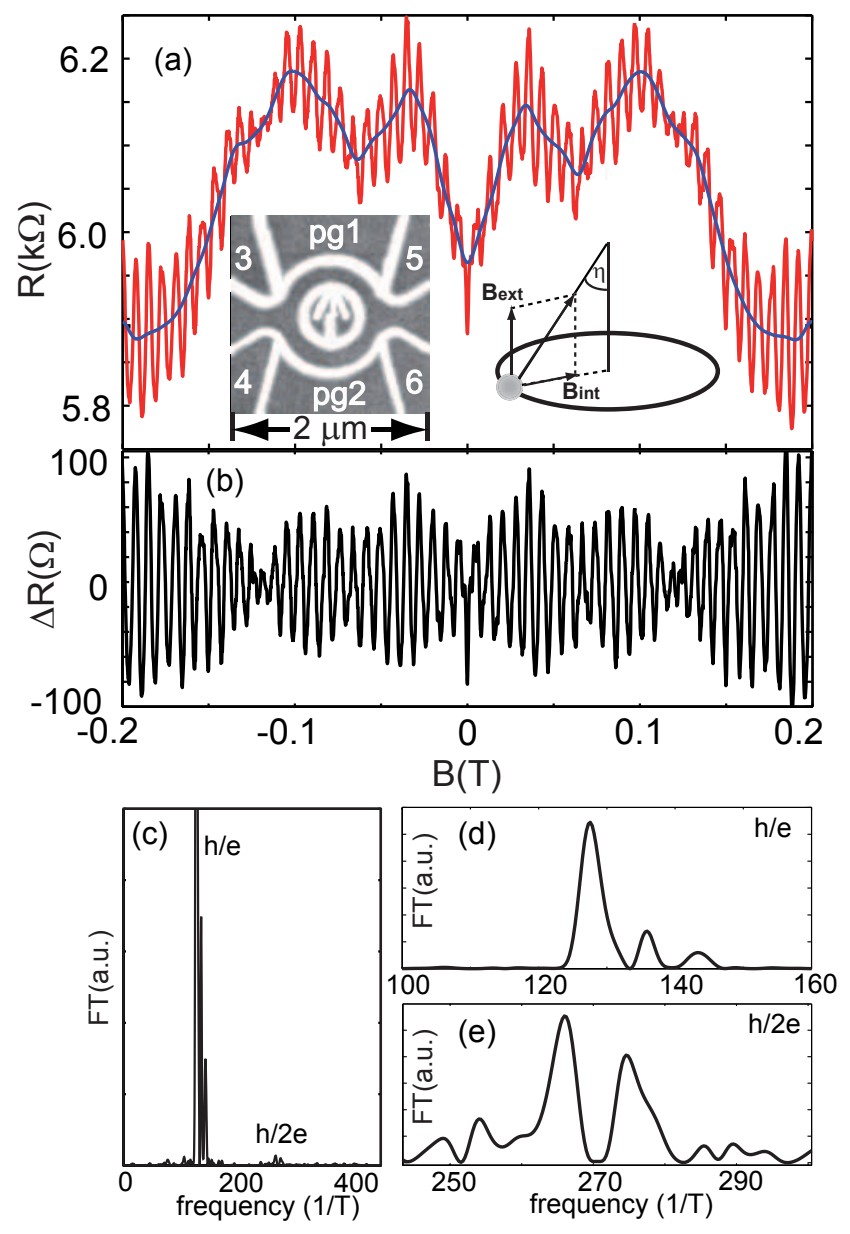

FIG. 1: (color online) (a) Measured magnetoresistance of the ring (strongly oscillating curve, red online) together with the low-frequency background resistance (smooth curve, blue online); Left inset: AFM micrograph of the ring with designations of the in-plane gates. Bright oxide lines fabricated by AFM oxidation lithography lead to insulating barriers in the 2DHG. Right inset: Scheme of a carrier travelling around the ring in the presence of the external field $\mathbf{B}_{\text {ext }}$ and SO induced intrinsic field $\mathbf{B}_{\text {int }}$. (b) AB oscillations obtained after subtraction of the low-frequency background from the raw data. A clear beating pattern is revealed in the $\mathrm{AB}$ oscillations. (c) Fourier transform spectra of the $\mathrm{AB}$ oscillations, revealing $h / e$ and $h / 2 e$ peaks. (d) Splitting of the $h / e$ Fourier peak. (e) Splitting of the $h / 2 e$ Fourier peak.

inating from the interference of time reversed paths, are expected to be more robust if the microscopic configuration of the arms is changed. Besides, $h / 2 e$ oscillations are less susceptible to the details how the spin rotates when it enters the ring than $h / e$ oscillations. This is due to the fact that the geometric phase accumulated along the paths contributing to the $h / 2 e$ oscillations is larger than that in the case of the $h / e$ oscillations and cannot be completely cancelled by the spin rotations in the contacts, as in the latter case [9]. In Fig. 1(c) we can identify 

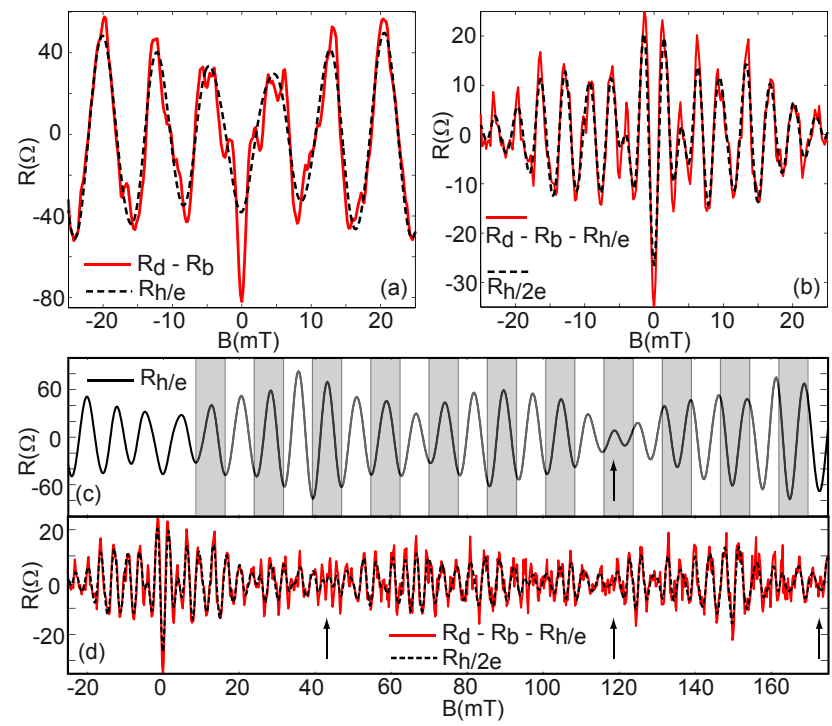

FIG. 2: (color online) (a) Measured magnetoresistance of the ring after subtracting the low-frequency background, $R_{d}-R_{b}$ (full line, red online), together with the filtered $h / e$ oscillations $R_{h / e}$ (dashed line). (b) Difference $R_{d}-R_{b}-R_{h / e}$ (full line red online) together with the inverse Fourier transform of the $h / 2 e$ peak $R_{h / 2 e}$ (dashed line). (c) Beating in filtered $h / e$ oscillations. The width of the gray and white rectangles corresponds to the period of $7.7 \mathrm{mT}$. The arrow points to the beating node where a phase jump of $\pi$ occurs. (d) Beating in filtered $h / 2 e$ oscillations with arrows indicating possible nodes.

the peak at about $270 \mathrm{~T}^{-1}$ in the Fourier spectrum, corresponding to $h / 2 e$ oscillations. If we zoom in on it [Fig. $1(\mathrm{e})]$, we see a splitting with the two main peaks having a separation of about $8 \mathrm{~T}^{-1}$, similar to the $h / e$ peak splitting. The splitting of the $h / 2 e$ Fourier peak arises due to the frequency shift of the main peak by $\pm 1 / B_{\text {int }}[5]$, and the obtained splitting of $8 \mathrm{~T}^{-1}$ allows to estimate the $\mathrm{SO}$ induced intrinsic field to be $B_{\text {int }} \approx 0.25 \mathrm{~T}$.

We now focus directly on the magnetic field-dependent resistance. In Fig. 2(a) we present the raw data after subtracting the low-frequency background (full line, red online) together with the filtered $h / e$ oscillations (dashed line) [21]. The $h / e$ contribution to the signal is the inverse Fourier transform of the $h / e$ peak in the Fourier spectrum. We will use the following notation below: $R_{d}$ denotes the raw data, $R_{b}$ is the low-frequency background, $R_{h / e}$ is the inverse Fourier transform of the $h / e$ peak and $R_{h / 2 e}$ is the inverse Fourier transform of the $h / 2 e$ peak in the Fourier spectrum. One can see in Fig. 2 (a) that $R_{d}-R_{b}$ contains additional resistance modulations, beyond the $h / e$ oscillations. In order to demonstrate that those additional features are due to $h / 2 e$ oscillations we plot in Fig. 2(b) the difference $R_{d}-R_{b}-R_{h / e}$ (full line, red online) and the curve $R_{h / 2 e}$ obtained by inverse Fourier transform of the $h / 2 e$ peak (dashed line) and find excellent agreement.
We further plot in Fig. 2(d) the difference $R_{d}-R_{b}-$ $R_{h / e}$ (full line, red online), together with the filtered $h / 2 e$ oscillations $R_{h / 2 e}$ (dashed line) in a larger range of magnetic fields. A beating-like behavior in the $h / 2 e$ oscillations is observed. Possible nodes develop around $40 \mathrm{mT}$, $115 \mathrm{mT}$, and $175 \mathrm{mT}$ [arrows in Fig. 2(d)]. The appearance of these unequally spaced nodes is in agreement with the complex split-peak pattern in Fig. 1(e). In the plot of the filtered $h / e$ oscillations [Fig. 2(c)] we notice that only the node around $115 \mathrm{mT}$ is common for both, $h / e$ and $h / 2 e$ oscillations, while the other two nodes in the $h / 2 e$ oscillations correspond to maxima in the beating of $h / e$ oscillations. This kind of aperiodic modulation of the envelope function of the $h / 2 e$ oscillations, rather than a regular beating, is predicted for the case of diffusive rings in the presence of Berry's phase [5], since the latter also changes with increasing external magnetic field.

The evolution of the $\mathrm{AB}$ oscillations upon changing plunger gate voltages $V_{p g 1}$ and $V_{p g 2}$ is explored in Fig. $3(\mathrm{a})$. Plunger gate voltages are changed antisymmetrically: $V_{p g 1}=-120 \mathrm{mV}-V ; V_{p g 2}=-120 \mathrm{mV}+V$. Two distinct features are visible: there is always a local minimum in the $\mathrm{AB}$ oscillations at $B=0 \mathrm{~T}$, and the oscillations experience a phase jump by $\pi$ around $V=27$ $\mathrm{mV}$. In order to understand the origin of these two features we analyze the filtered $h / e$ (not shown) and $h / 2 e$ oscillations [Fig. 3(b)] as a function of $V$. The $h / e$ oscillations experience a phase jump of $\pi$, while the $h / 2 e$ oscillations do not [Fig. 3(b)]. We have explored this behavior in several other gate configurations and always found the same result. The reason for such a behavior is that the $h / e$ oscillations are sensitive to the phase difference $\Delta \varphi=k_{1} l_{1}-k_{2} l_{2}$ between the two arms, which can be changed by the plunger gates, while the AAS $h / 2 e$ oscillations are not. We observe a resistance minimum at $B=0 \mathrm{~T}$ for all gate configurations [Fig. 3(a)], which is due to a minimum at $B=0 \mathrm{~T}$ in the $h / 2 e$ oscillations [Fig. 3(b)]. It indicates that time reversed paths of the holes' spinors interfere destructively due to strong SOI, in contrast to n-type GaAs systems where $h / 2 e$ oscillations produce a resistance maximum at $B=0 \mathrm{~T}$ [22]. This effect has the same origin as the weak anti-localization (WAL) effect. However, the observed minimum is not caused by WAL in the ring leads, since the WAL dip in bulk 2D samples has a much smaller magnitude (less than $1 \Omega,[19]$ ) than the minimum at $B=0 \mathrm{~T}$ in the ring. The resistance minimum at $B=0 \mathrm{~T}$ is a result of the destructive interference of the holes' spins in the ring.

The adiabatic regime is reached when $\omega_{B} / \omega_{\text {orbit }}>>1$, where $\omega_{B}=g \mu_{B} B_{t o t} / 2 \hbar$ is the spin precession frequency around $\mathbf{B}_{\text {tot }}$, while $\omega_{\text {orbit }}=v_{F} / r$ is the orbital frequency of the holes around the ring in the ballistic regime. ptype GaAs systems have strong SOI, and therefore large $B_{\text {int }}$, which, together with a small $v_{F}$ (due to the large effective mass of the holes) makes p-types systems very favorable for reaching the adiabatic regime compared to other systems. Using the estimated value for $B_{\text {int }}$ of $0.25 \mathrm{~T}$ and assuming a holes' $g$ factor of 2 , we obtain 


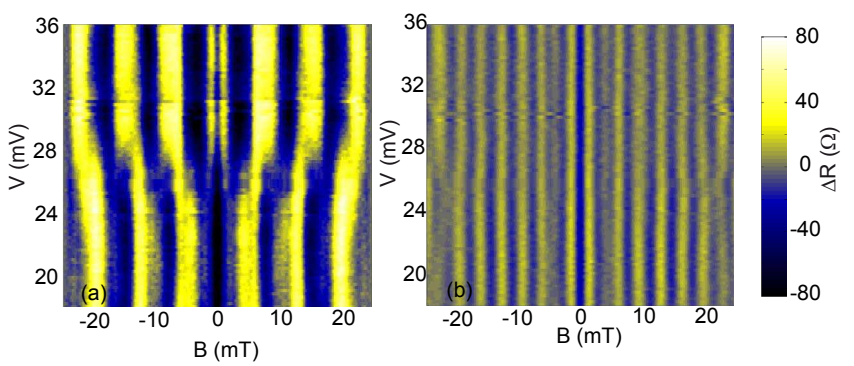

FIG. 3: (color online) (a) Evolution of the AB oscillations upon changing the plunger gate voltages $V_{p g 1}=-120 \mathrm{mV}$ $-V ; V_{p g 2}=-120 \mathrm{mV}+V$. (b) Filtered $h / 2 e$ oscillations as a function of the plunger gate voltages, showing the local minimum at $B=0 \mathrm{~T}$ at all gate voltages.

$\omega_{B} / \omega_{\text {orbit }} \approx 0.2-0.3$ for the measured range of $B_{\text {ext }}$ up to $0.2 \mathrm{~T}$. Therefore the adiabatic regime is not fully reached in our measurements.

There remains a pronounced discrepancy between the internal magnetic field obtained from the beating of the $\mathrm{SdH}$ oscillations of $7 \mathrm{~T}$ (converting the corresponding energy scale $\Delta_{S O} \approx 0.8 \mathrm{meV}$ to a magnetic field via the Zeeman splitting) and the field scale of $0.25 \mathrm{~T}$ obtained from the beating of the $\mathrm{AB}$ oscillations. The latter evaluation is strictly valid in the diffusive regime [5] while our sample is at the crossover to the ballistic regime. It is also not clear how the limited adiabaticity in our samples will influence these numbers.

In a straightforward picture one would expect that the node of the beating in the $h / 2 e$ oscillations occurs at half the magnetic field as the node in the $h / e$ oscillations since the accumulated phase difference between the two spin species should be proportional to the path length travelled in the ring. Within experimental accuracy the data in Fig. 1 (d) and (e) suggests that the splitting in the corresponding Fourier transforms is the same.

In conclusion, we have measured Aharonov-Bohm oscillations in a ring defined on a 2D hole gas with strong spin-orbit interactions. We observe a beating in the measured resistance which arises from an interplay between the orbital Aharonov-Bohm and a spin-orbit induced geometric phase. In addition we resolve $h / 2 e$ oscillations in the ring resistance, and find that they also show a beating-like behavior, which produces a splitting of the $h / 2 e$ peak in the Fourier spectrum. A resistance minimum at $B=0$, present in all in-plane gate configurations, demonstrates the destructive interference of the hole spins propagating along time reversed paths.

We thank Daniel Loss and Yigal Meir for stimulating discussions. Financial support from the Swiss National Science Foundation is gratefully acknowledged.
[1] Y. Aharonov and D. Bohm, Phys. Rev. 115, 485 (1959)

[2] M.V. Berry, Proc. R. Soc. A 392, 45 (1984)

[3] Y. Aharonov and J. Anandan, Phys. Rev. Lett. 58, 1593 (1987)

[4] D. Loss, H. Schoeller, P.M. Goldbart Phys. Rev. B 59, 13328 (1999).

[5] H.A. Engel and D. Loss, Phys. Rev. B 62, 10238 (2000)

[6] Y. Meir, Y. Gefen and O. Entin-Wohlman, Phys. Rev. Lett. 63, 798 (1989); A.G. Aronov and Y.B LyandaGeller, Phys. Rev. Lett. 70, 343 (1993); H. Mathur and A.D. Stone, Phys. Rev. Lett. 68, 2964 (1992); T. Z. Qian and Z.B. Su, Phys. Rev. Lett. 72, 2311 (1994); J. Nitta, F.E. Meijer, H. Takayanagi, Appl. Phys. Lett. 75, 695 (1999); D. Frustaglia and K. Richter, Phys. Rev. B 69, 235310 (2004).

[7] A. F. Morpurgo, J. P. Heida, T. M. Klapwijk, B. J. van Wees, and G. Borghs, Phys. Rev. Lett. 80, 1050 (1998).

[8] J.B. Yau, E.P. De Poortere and M. Shayegan, Phys. Rev. Lett. 88, 146801 (2002).

[9] M.J. Yang, C.H. Yang, Y.B. Lyanda-Geller, Europhys. Lett. 66, 826 (2004).

[10] M. König et al., Phys. Rev. Lett. 96, 076804 (2006)

[11] T. Bergsten, T. Kobayashi, Y. Sekine, and J. Nitta, Phys. Rev. Lett. 97, 196803 (2006)

[12] B. Habib, E. Tutuc, and M. Shayegan, Appl. Phys. Lett. 90, 152104 (2007).
[13] R. Winkler, Spin-Orbit Coupling Effects in TwoDimensional Electron and Hole Systems, Springer Tracts in Modern Physics, Volume 191, Springer-Verlag (2003)

[14] A. Stern, Phys. Rev. Lett. 68, 1022 (1992)

[15] A. G. Mal'shukov, V. V. Shlyapin, and K. A. Chao, Phys. Rev. B 60, R2161 (1999)

[16] H. De Raedt, Phys. Rev. Lett. 83, 1700 (1999); A. F. Morpurgo, J. P. Heida, T. M. Klapwijk, B. J. van Wees, and G. Borghs, Phys. Rev. Lett. 83, 1701 (1999)

[17] A. G. Mal'shukov and K. A. Chao, Phys. Rev. Lett. 90, 179701 (2003); J. B. Yau, E. P. De Poortere, and M. Shayegan, Phys. Rev. Lett. 90, 179702 (2003); A. G. Wagh and V. C. Rakhecha, Phys. Rev. Lett. 90, 119703 (2003); J. B. Yau, E. P. De Poortere, and M. Shayegan, Phys. Rev. Lett. 90, 119704 (2003)

[18] B. Grbić, R. Leturcq, K. Ensslin, D. Reuter, and A.D. Wieck Appl. Phys. Lett. 87, 232108 (2005)

[19] Boris Grbić, PhD thesis No. 17248, ETH Zurich (2007).

[20] J. P. Lu et al., Phys. Rev. Lett. 81, 1282 (1998)

[21] M. Sigrist et al., Phys. Rev. Lett. 93, 066802 (2004).

[22] T. Ihn et al., Advances in Solid State Physics Vol. 43 eddited by B. Kramer (Springer-Verlag, New York, 2003) p. 139; A. E. Hansen, A. Kristensen, S. Pedersen, C. B. Sørensen, and P. E. Lindelof, Phys. Rev. B 64, 045327 (2001). 